\title{
SIMULATION AND CHARACTERIZATION OF RANDOM SYSTEMS OF HARD PARTICLES
}

\author{
DIETRICH STOYAN \\ Institut für Stochastik, TU Bergakademie Freiberg, 09596 Freiberg, Germany \\ e-mail: stoyan@orion.hrz.tu-freiberg.de \\ (Accepted May 4, 2002)
}

\begin{abstract}
This paper surveys methods for the simulation of random systems of hard particles, namely sedimentation and collective rearrangement algorithms, molecular dynamics, and Monte Carlo methods such as the MetropolisHastings algorithm. Furthermore, some set-theoretic statistical characteristics are discussed: the covariance and topological descriptors such as specific connectivity numbers and Mecke's morphological functions.
\end{abstract}

Keywords: connectivity number, covariance, Gibbs process, morphological function, packing, porous medium, random set, simulation.

\section{INTRODUCTION}

In many situations engineers and many kinds of scientists are confronted with irregular spatial structures which can be interpreted and modeled as systems of hard particles, in particular packings. Examples are structures which result from packing processes (such as sinter metals), materials such as concrete (with the sand grains as 'particles'), porous media like sand stone or soil, granular matter like sand and systems of cell nuclei. It is important to investigate statistically and stereologically the geometry of these structures, since there are in general close relationships between geometrical structure and bulk material properties such as mechanical strength or conductivity for electric current and fluids. However, it is rather difficult to fit models to such structures since for dense and packed structures until now there are no tractable mathematical models for which formulas for the main geometrical characteristics are known. Therefore, the only way for mathematical-statistical investigations of such systems is computer simulation. Such simulations are a pragmatic way to obtain quantitative results. They are difficult and have been made mainly only for spherical and ellipsoidal particles.

The main aim of this paper is to describe some of the simulation methods used in the context of hard particle systems. Additionally, the use of the covariance and of topological characteristics such as specific connectivity number and Klaus Mecke's morphological functions is discussed.

\section{GENERAL IDEAS FOR THE SIMULATION OF RANDOM SYSTEMS OF HARD PARTICLES}

There are two main classes of random systems of hard particles:

- systems in which the particles are usually isolated, where contacts are only rare events (as in the case of sand grains in concrete or biological cell nuclei) and

- systems of packed particles where the particles are in contact and may even form a mechanically stable structure.

In this paper such systems are modelled as infinite structures, which are statistically homogeneous or, in other words, stationary and isotropic.

In the first case, Gibbs processes are a probably good model. In contrast, for random packings there is until now no rigorous mathematical model, even in the case of identical spheres; there are only various simulation algorithms.

Therefore, many existing studies of random sphere systems are not much more than studies of the properties of structures obtained by various simulation algorithms. All these algorithms can be interpreted as simulations of Markov chains, which are either homogeneous and heterogeneous.

A Markov chain is a particular stochastic process with the lack-of-memory property, where a new state is randomly generated from the present one, independently of past states. In the given case, the states of the process are configurations of sphere systems, i.e. the process is rather complicated. A very 
good reference to Markov chain Monte Carlo is the book Gilks et al. (1996).

In the case of non-spherical particles, all is still more complicated since there are different physical packing conditions. For example, packings of cylinders generated in air and viscous fluid show remarkable differences in density and structure.

\section{THE RSA MODEL}

Sometimes engineers have used spontaneously a quite simple procedure to obtain random systems of hard spheres: the SSI model or the RSA model ('simple sequential inhibition model', 'random sequential absorption model'). Usually, it is defined in a bounded region $B$ of three-dimensional space. Spheres are placed sequentially and randomly in $B$. If a new sphere is placed so in $B$ that it intersects a sphere already existing then the new sphere is rejected and a new placing trial is made. The process of placing spheres is stopped when the system is in its 'jamming limit', when all placing attempts must fail. Clearly, there are no contacts between the spheres.

If the sphere diameters are not constant, then smaller spheres have greater chances to find a position in $B$ than larger ones if the diameters used in the placing attempts are chosen randomly. Thus it is necessary to discriminate between the sphere diameter distribution in the placing trials and in the final RSA model, see the discussion in Stoyan and Schlather (2000).

It is possible to define an analogous stationary model, which is defined in the whole $\mathbb{R}^{3}$, see Stoyan and Schlather (2000). Its volume fraction $V_{V}$ is only 0.382 in the case of equal spheres. This value is too small in many practical situations. Nevertheless, this model is of great physical relevance, see Evans (1993). Döge (2001) has developed a clever method for the efficient simulation of the RSA model.

\section{SEDIMENTATION ALGORITHMS}

Sphere systems which are closer to true packings are obtained by the sedimentation algorithm of Jodrey and Tory (1979). Variations of this algorithm are described in Jodrey and Tory $(1979,1985)$ and Tory et al. (1973). It generates a system of spheres in a parallelepipedal container; periodic boundary conditions try to ensure that the simulated sphere system can be considered as a sample of a statistically homogeneous structure, if the container is large enough. A typical implementation of the sequential addition concept is as follows. First, some initial configuration of $n_{0}$ spheres is produced, usually a layer of spheres at the bottom of the container. Every subsequent iteration drops a new sphere into the system. The sphere then moves in a prescribed direction (typically downwards, following gravitation) until it hits another already existing sphere in the system. Then, the sphere rolls along others until it reaches a stable configuration (typically, if it is in contact with three supporting spheres). If a stable configuration cannot be found after long time, the algorithm stops or repeats the trial with a new sphere. This process of filling the container with new spheres continues until all planned spheres are packed or if the container is filled up. Typically, the packings obtained by this method are not very dense, since there is no additional pressing or densification procedure; in the case of identical spheres, the obtained volume fraction $V_{V}$ is about 0.58 , which is a little below the value for a natural so-called random loose packing.

Probably the idea of this algorithm has not yet been applied to the case of non-spherical particles. Probably this generalization is difficult and one has to expect that without a densification procedure such packings are only of a very poor quality.

\section{COLLECTIVE REARRANGEMENT ALGORITHMS}

A family of algorithms which produce 'better' packings, i.e. packings with higher volume fractions, are algorithms sometimes called 'collective rearrangement' algorithms. Here the number of spheres $N$ is fixed during the whole simulation and overlappings are occasionally permitted. During the simulation they are reduced by moving the spheres and by shrinking of their diameters. The oldest algorithm of this type is probably Stillinger's algorithm, see Stillinger et al. (1964). In the present paper the packing algorithm developed by Moscinski and Bargieł and colleagues, see Moscinski et al. (1989) and Bargieł and Moscinski (1991), is briefly described. It was also used to produce Figs. 1 and 2 in Bezrukov et al. (2001). This algorithm, called 'force biased algorithm', is able to produce packings with a wide range of densities up to ultradense packings with $V_{V}$ greater than 0.70 in the case of identical spheres, see Bargieł and Tory (1993).

The initial configuration of the algorithm is a set of $N$ spheres $b\left(x_{i}, r_{i}\right)$ with centres $x_{i}$ uniformly distributed in the given parallelepipedal container and radii $r_{i}$ chosen according to a prescribed radius distribution function. Overlappings are permitted. While the number of spheres is fixed, the algorithm attempts to reduce overlaps between spheres by pushing apart overlapping spheres and gradual shrinking of the radii. In this algorithm only the starting configuration is random; the rest is completely deterministic. 
The algorithm defines a 'repulsion force', $F_{i j}$, between each pair $(i, j)$ of overlapping spheres as

$$
F_{i j}=\rho \mathbf{1}_{i j} p_{i j} \frac{x_{j}-x_{i}}{\left|x_{j}-x_{i}\right|},
$$

where $p_{i j}$ is a given 'potential' function, $\rho$ is a scaling factor, and $\mathbf{1}_{i j}$ is equal to 0 if $b\left(x_{i}, r_{i}\right) \Gamma b\left(x_{j}, r_{j}\right)=\emptyset$ and equal to 1 otherwise.

The new position of the $i$-th sphere is given by

$$
x_{i}:=x_{i}+\frac{1}{2 r_{i}} \sum_{j \neq i} F_{i j} .
$$

In every step of the algorithm this shifting operation and a shrinking operation are performed for all spheres until all overlappings vanish. This shrinking is organized in such a way that the absolute values of radii may decrease but their proportions are not changed during the simulation.

The choice of $p_{i j}$ is a non-trivial task but crucial to the efficiency of the algorithm. For the case of equal spheres (i.e. constant radii) the potential function may be simply proportional to the volume of intersection of the overlapping spheres $i$ and $j$. However, for radius distributions leading to large differences of sphere radii this potential is ineffective. The paper Bezrukov et al. (2002) describes a better potential and the way in which the diameters are shrinked in the algorithm.

Probably, the force-biased algorithm has not yet been generalized to the non-spherical case. But such generalization should be possible and the chances to obtain packings of a high density are perhaps better than in the case of sedimentation algorithms.

\section{MOLECULAR DYNAMICS}

A further family of algorithms for obtaining dence random systems (which can be used as approximations for packings), which is very popular among physicists and engineers, is molecular dynamics invented by Alder and Wainwright (1960). A today well developed method is the distinct-element method (DEM) introduced by Cundall (1971) for the analysis of rock-mechanical problems and further developed until commercially used programs, see Cundall and Hart (1992). Here the particles move in large containers following Newton's laws of motion, having contacts with other particles and the container's boundaries. The calculations are performed in discrete time steps, where in each step the set of contacts is updated, then the contact forces are determined applying a force-displacement law, and finally for each particle its new velocity and position is calculated. States of equlilibrium are obtained when the internal forces balance. The particles are either spheres or polyhedra.

\section{HARD-CORE GIBBS PROCESSES}

An important mathematical model for systems of non-overlapping particles is the hard-core Gibbs process, see Stoyan et al. (1995) for explanation both for the finite and infinite, stationary case. The finite hard-core Gibbs process can be explained as follows. There is a bounded region in space $W$, for example a parallelepiped. In $W$ a point process is given, where the points serve as 'centres' of hard particles. If the number of points is fixed (equal to $n$, 'canonical' case) and only their positions are random and all particles are of the same shape and orientation and size $K$ (i.e., the particle centred at $x$ is $K+x$ ), then the joint probability density function $f$ of the points is given as follows:

$$
f\left(x_{1}, \ldots, x_{n}\right)=Z \exp \left\{-\sum_{i<j} d\left(x_{i}, x_{j}\right)\right\},
$$

where

$$
d\left(x_{i}, x_{j}\right)= \begin{cases}0 & \text { if } K+x_{i} \cap K+x_{j}=\emptyset \\ \infty & \text { otherwise }\end{cases}
$$

and $Z$ is the necessary normalizing constant making $f$ to a probability density.

Generalization to the case of a random number of particles ('grand canonical' case), and even to infinite, stationary systems with particles of random orientation and size is possible. From a rather abstract mathematical standpoint, the distribution given by (1) can be interpreted as the uniform distribution on the set of all configura- tions of hard particles with centre in $W$. This may explain why there are so many different simulation algorithms for obtaining samples of hardcore Gibbs processes.

Typically, hard-core Gibbs processes are not packings in the narrow sense, since between the particles there are usually (small) gaps; but for densities high enough, they are good approximations for packings. The volume fraction $V_{V}$ of stationary hard-core Gibbs processes of identical spheres can take all values between 0 und the highest value $\pi / \sqrt{18}=$ 0.74 , which is obtained for the densest deterministic packing.

In simulations of samples of stationary Gibbs processes, structures in finite regions are generated, usually in parallelepids. In order to come close to the situation of samples from a statistically homogeneous process, periodic boundary conditions are used. Various simulation methods can be used for the simulation of such finite Gibbs processes, see 
Binder (1997, 2001). A very popular possibility is also here molecular dynamics, see e.g. Truskett et al. (1999). This method has been also applied to the case of ellipsoidal particles, see e.g. Schmid (2002) and Figs. 1 and 2.

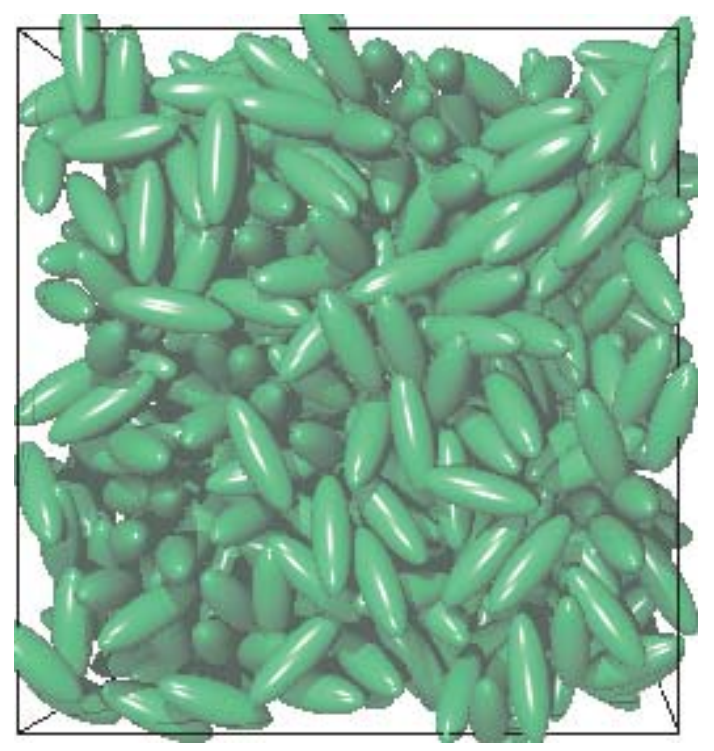

Fig. 1. A simulated sample of a Gibbs process of hard ellipsoids in case of low density, $V_{V}=0.38$ (Courtesy of Nguyen Hoang Phuong, Bielefeld).

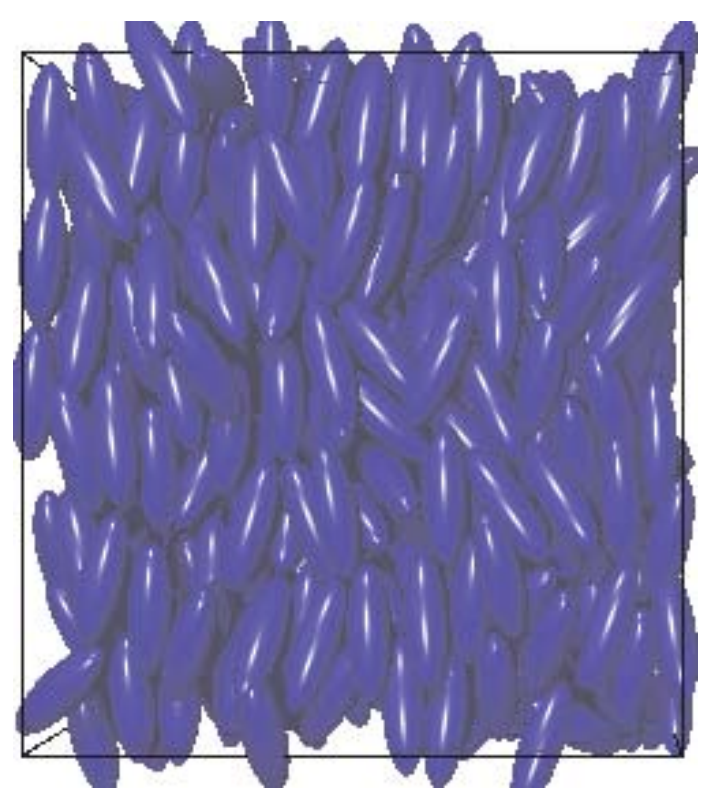

Fig. 2. A simulated sample of a Gibbs process of hard ellipsoids in case of $V_{V}=0.47$. Observe the parallelity tendency in the structure (Courtesy of Nguyen Hoang Phuong, Bielefeld).
But there are also other methods for the simulation of Gibbs processes, which use other, perhaps more efficient concepts of Markov chain Monte Carlo. The classical algorithm is the Metropolis-Hastings algorithm, Metropolis et al. (1953), Hastings (1970) and Binder $(1997,2001)$. The idea is to construct a Markov chain with states in the set of all possible configurations of systems of hard spheres, with the property that the stationary distribution of the chain is just a Gibbs distribution such as (1). Then the Markov chain is simulated for a long time in the hope to get to the stationary state. There samples are taken which are considered as samples of the Gibbs process. In the case of a grand canonical system of hard spheres in a region $W$, typically a parallelepiped, this algorithm works as follows, see Mase et al. (2001). For a simpler version which is closer to the original algorithm by Metropolis, see Torquato (2002), p. 275.

Assume that a current state $\varphi=\left\{x_{1}, x_{2}, \ldots, x_{n}\right\}$ of the sphere system is given. It is then proposed to either (a) insert, (b) delete, or (c) move a sphere with probabilities $p^{i n s}$, $p^{d e l}$ and $1-p^{i n s}-p^{d e l}$, respectively. The proposal for the next state in the chain is taken as follows:

(a) old configuration $\varphi$ plus a new point $x$ which is sampled in $W$ from a density $b(\varphi,$.$) ,$

(b) old configuration $\varphi$ minus one of its points with probability $d(\varphi,$.$) ,$

(c) old configuration minus one of its points (chosen with probability $d(\varphi,).), x$, plus a new point sampled from a density $m(\varphi, x,$.$) .$

Possible choices of the probabilities and densities are: $p^{i n s}=p^{d e l}=p$ with $0 \leq p \leq 0.5, b(\varphi,$.$) is$ independently of $\varphi$ the uniform distribution on $W$, $d(\varphi,$.$) is the discrete uniform distribution on \varphi$, and $m(\varphi, x,$.$) is the uniform distribution on the cube of$ side length $2 \varepsilon$ centered at $x$ and sides parallel to the coordinate axes. The choice of $p$ and $\varepsilon$ is usually made after some experiments, see Mase et al. (2001).

In the Metropolis-Hastings algorithm these proposals are accepted with some probability, called Hastings ratio; in case of rejection of the proposal, the chain remains in its given state $\varphi$. This acceptance rule ensures that the chain has the equilibrium or stationary density $f$.

The Hastings ratios depend on the type of transition and are given by

$$
\text { (a) } \frac{f\left(\varphi^{\prime}\right) p^{\text {del }} d\left(\varphi^{\prime}, x\right)}{f(\varphi) p^{\text {ins }} b(\varphi, x)}
$$


(b)

$$
\frac{f\left(\varphi^{\prime}\right) p^{i n s} b\left(\varphi^{\prime}, x\right)}{f(\varphi) p^{d e l} d(\varphi, x)}
$$

(c) $\frac{f\left(\varphi^{\prime}\right) d\left(\varphi^{\prime}, y\right) m(\varphi \backslash\{x\}, y, x)}{f(\varphi) d(\varphi, x) m(\varphi \backslash\{x\}, x ; y)}$

where $\varphi^{\prime}$ denotes the proposed configuration.

The sequence of states $\varphi$ forms a homogeneous ergodic Markov chain the stationary distribution of which has the probability density $f$. Consequently, after a sufficient long 'burning-in time' the states of the sequence can be taken as samples from the Gibbs process.

The Metropolis-Hastings algorithm was again refined in Mase et al. (2001) in order to simulate very dense systems of discs and to investigate the so-called melting transition of hard discs in two dimensions. The new algorithm is called 'simulated tempering' (not 'simulated annealing') and goes back to Marinari and Parisi (1992). The idea is to consider a Markov chain the states of which are not only particle configurations, but instead pairs \{particle configuration, temperature with a discrete series of temperatures. Clearly, this new chain has a much larger state space, but it has a better mixing behaviour than the corresponding MetropolisHastings chain. Thus it can be still successfully simulated in situations where the Metroplis-Hastings algorithm converges unacceptably slowly and yields highly correlated results.

For fixed temperature the Markov chain behaves like a Metropolis-Hastings chain as explained above. But with increasing temperature overlappings of particles are permitted in increasing extent, while for the coldest temperature such overlappings are completely forbidden. The transitions in the new Markov chain are organized in such a way that samples taken in the cold state can be considered as samples from the hard core Gibbs process to be studied.

\section{A REMARK ON THE CASE OF NON-SPHERICAL PARTICLES}

The simulation of random dense systems or packings of non-spherical particles is still a very difficult task. As mentioned above, there are simulations for polyhedral and ellipsoidal particles, but their quality as models for packings has not been systematically studied. In this case even $V_{V}$ depends strongly on both particle shape and packing method (characterized by the degree of tapping and pressing).

\section{STATISTICAL CHARACTERIZATION BY THE COVARIANCE}

A very valuable statistical quantity also for systems of hard particles is the covariance $C(r)$. It has the advantage that it can be determined very easily stereologically, see Stoyan et al. (1995). By some training the statistician is able to make similar use of $C(r)$ as of the more explicit pair correlation function. Note that Stoyan and Stoyan (1994) explains in detail the interpretation of empirical pair correlation functions. This helps also to interpret the curves in Fig. 3, if they are compared with Figs. 4 and 5 in Bezrukov et al. (2001).

The covariance is the probability that both members of a random point pair of distance $r$ fall into the union set of all spheres, which is denoted by $X$. Because of stationarity and isotropy, this probability can be written as

$$
C(r)=P(o \in X, \mathbf{r} \in X) \quad \text { for } \quad r \geq 0
$$

where $o$ is the origin of the space and $\mathbf{r}$ is a point of distance $r$ from $o$. (Because the structure is assumed to be statistically homogeneous, any point pair of distance $r$ can be used.) It is useful to decompose $C(r)$ as the sum of two terms

$$
C(r)=C_{i}(r)+C_{o}(r) \quad \text { for } \quad r \geq 0 .
$$

Here the first term is the probability that the two points belong to the same particle and the second the probablity that the points belong to different particles, see Torquato (1991). The first term depends only on particle size and shape and can be computed separately, given the probability distribution of the particles. It is

$$
C_{i}(r)=N_{V} \bar{\gamma}(r)
$$

where $\bar{\gamma}(r)$ is the mean isotropized set covariance of the typical particle and $N_{V}$ the mean number of particles per volume unit. The paper by Delarue and Jeulin (2001) demonstrates the use of the covariance, in particular of $C_{o}(r)$, in the study of packings of spheres. Fig. 3 shows $C_{o}(r)$ for three different packings. These differences demonstrate that $C_{o}(r)$ is indeed a valuable characteristic, since shows large differences for the three packings. In particular, the great variablity of the lognormal packing is expressed by the very smooth form of $C_{o}(r)$. 


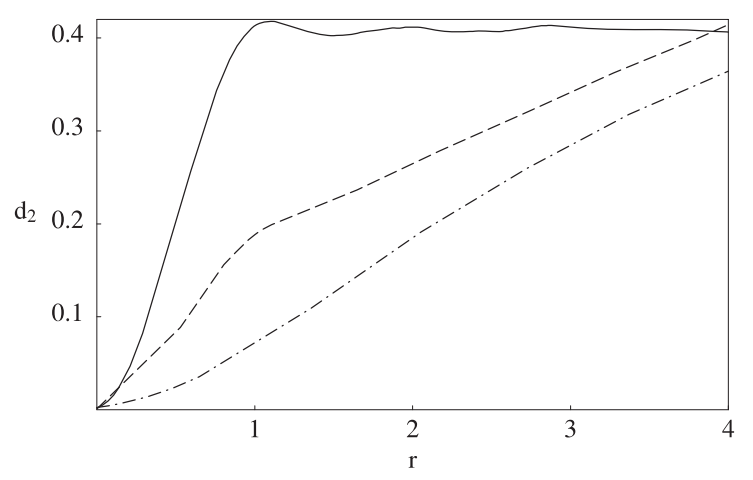

Fig. 3. The covariance functions $C_{o}(r)$ for three different packings of spheres.

- : identical spheres, $V_{V}=0.64, \quad-\cdot-$ : lognormal diameter distribution, $V_{V}=0.68$,

_ - : binary mixture with diameters $1(90 \%)$ and 10 $(10 \%), V_{V}=0.82$.

\section{SPECIFIC CONNECTIVITY NUMBER}

The specific connectivity number $K_{V}$ is a topological characteristic. If all particles are convex and isolated (i.e., there are no contacts between them) then $K_{V}$ is the same as mean particle number per unit volume, $N_{V}$. But if the particles have contacts, then $K_{V}$ gives valuable information on the topology of the particle system; even negative values of $K_{V}$ are possible.

The specific connectivity number $K_{V}$ (or density of Euler characteristic) can be explained starting from the Euler-Poincare characteristic. The Euler-Poincaré characteristic $\chi$ is a generalization of number of components' for nonconvex sets and is a topological invariant, i.e., it is not changed if the set is continuously and biuniquely deformed. For a polyconvex set $K$, i.e. a finite union of compact convex sets $K_{1}, \ldots, K_{n}$, it is

$$
\begin{aligned}
\chi(K)=\sum_{i=1}^{n} \chi\left(K_{i}\right)-\sum_{i=1}^{n} \sum_{j=i+1}^{n} \chi\left(K_{i}\left\ulcorner K_{j}\right)+\cdots\right. \\
+(-1)^{n-1} \chi\left(\bigcap_{i=1}^{n} K_{i}\right),
\end{aligned}
$$

where $\chi(K)=1$ for a convex set $K$. (Note that the intersection of convex sets is again a convex set, so that the right hand side is well defined. In its third term, intersections of three sets appear.) Thus for a single sphere $\chi(K)=1$, while for a set formed by the union of four identical spheres arranged in a chain .... and touching neighbours, so that the two inner spheres contact two other spheres, it is

$$
\chi(K)=4-3=1,
$$

and for six spheres arranged as ::: in two parallel touching chains, where the two inner spheres contact three other spheres,

$$
\chi(K)=6-7=-1 .
$$

The specific connectivity number of a stationary random closed set is given by

$$
K_{V}=E\left(\chi \left(X\ulcorner W)-\chi\left(X\left\ulcorner\partial^{+} W\right)\right) ;\right.\right.
$$

i.e. the mean of the Euler-Poincaré characteristic of $X \cap W$ reduced by a boundary-related term, where $W$ is a cube of volume 1 and $\partial^{+} W$ is the 'upper right boundary of $W^{\prime}, \partial^{+}=\left\{x=\left(x_{1}, x_{2}, x_{3}\right): \max x_{i}=1\right\}$. In the particular case of a packing of spheres it is

$$
K_{V}=N_{V}\left(1-\frac{c}{2}\right)
$$

where $N_{V}$ is the number of spheres per volume unit and $c$ is the mean number of spheres in contact with a typical sphere of the packing, see Mecke and Stoyan (2001).

\section{MECKE'S MORPHOLOGICAL FUNCTIONS}

In a series of papers, K. Mecke developed a powerful technique for the topological characterization of structures which can be described by stationary random sets, see Mecke and Wagner (1991) and Mecke (2000). He speaks about Minkowski functionals or functions, but perhaps the term 'morphological functions' is a better general notation. The idea is a natural generalization of the well-known spherical contact distribution $H_{s}(r)$, see Stoyan et al. (1995). One of its definitions for a statistically homogeneous random set $X$ is as follows. Let $X_{r}$ be the set $X$ dilated by the sphere $b(o, r)$ of radius $r$ centred at the origin $o$, i.e., $X_{r}=X \oplus b(o, r)$ is the set of all points of the space which belong to $X$ or have a distance less than $r$ from $X$. Let $V_{V}(r)$ be the volume fraction of $X_{r}$ and $V_{V}(0)=V_{V}$ that of $X$. Then

$$
H_{s}(r)=1-\frac{1-V_{V}(r)}{1-V_{V}} \quad \text { for } \quad r \geq 0 .
$$

This function describes statistically the pore space between the spheres; it can be also interpreted as the distribution function of the distance from a random test point in pore space to the nearest surface point. Formula (4) is usually the base for the statistical determination of $H_{s}(r)$ in image analysis, since pixel or voxel counting and dilation of pixel sets are basic operations in 2D and 3D image analysis. 
Consider now the specific surface (or the Minkowski functional of order 1) of $X_{r}$. This is a function $S_{V}(r)$ of $r$. The same can be done with the other Minkowski functionals, e.g. with the EulerPoincaré characteristic, which leads to the function $K_{V}(r)$, the specific connectivity number of $X_{r}$. So the statistician has four functions of $r$, which describe various geometrical properties of the set $X$. While $V_{V}(r)$ and $H_{s}(r)$ tell frequently not very much about the morphology of $X$, the ensemble of all four functions together enables a very instructive understanding. In particular, $K_{V}(r)$ is often very interesting. By the way, also negative values of $r$ can be used; for negative $r$ the set $X \in b(o, r)$ is considered, the 'erosion' of $X$. Instead of dilation and erosion also the more complex morphological operations closing and opening can be used.

Fig. 4 shows these functions for two packings of spheres. The curves for $V_{V}(r)$ differ only a little. Because of discretization effects, the value for $r=0$ for the lognormal case is a bit too small. However, the qualitative behaviour is correctly shown. $R$ is the (mean) sphere radius. Since there are many small spheres in the lognormal case, $S_{V}(r) R$ is very large for small $r$. Clearly, $S_{V}(-R)=0$ for the case of identical spheres. The mean curvature density $M_{V}(r)$ is multiplied by $R^{2}$ in order to obtain a dimensionfree characteristic. $M_{V}(r)$ must linearly decrease for negative $r$ in the case of identical spheres. It becomes negative since for positive $r$ the spheres overlap and sharp non-convex edges appear. The small spheres in the lognormal case cause big differences to the case of identical spheres. The specific connectivity number $K_{V}(r)$ is multiplied by $V / N=$ volume/sphere number. $K_{V}(r) V / N$ is equal to one for negative $r$ (if $r / R \leq 1$ ) for identical spheres. For $r=0$ it is negative because of (3). With increasing positive $r$ it tends towards zero. It is typical that the morphological differences are best visible for $M_{V}(r)$ and $K_{V}(r)$.

\section{CONCLUSIONS}

The art of simulating systems of hard particles has made big progress. In near future we can expect the appearance of high-quality commercial programs. Also the case of non-spherical particles will be attacked. This opens the way to statistical analyses of real geometrical structures of very high complexity.

In such analyses not only point process methods (in particular the pair correlation function) should be used, but also the characteristics discussed in the present paper. They are easier to measure, in part they can be even obtained from planar sections.
For mathematicians there is the challenge to develop formulas for the characteristics of the systems, which can today be only simulated.
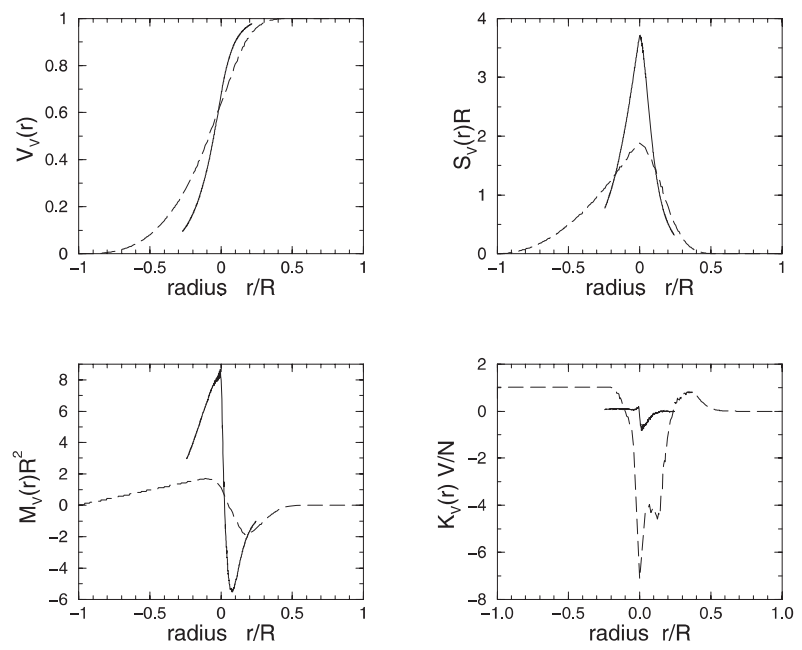

Fig. 4. K. Mecke's morphological functions, for a packing of identical spheres with $V_{V}=0.64$ and for a packing of spheres with a lognormal radius distribution (-). See the text for more explanation.

\section{REFERENCES}

Alder BJ, Wainwright TE (1960). Studies in molecular dynamics. II. Behaviour of a small number of elastic spheres. J Chem Phys 33:1439-51.

Bargiel M, Moscinski J (1991). C language program for the irregular packing of hard spheres. Computer Phys Comm 64:183-92.

Bargiel M, Tory EM (1993). Packing fraction and measures of disorder of ultradense packings of equal spheres. I. Nearly ordered packing. Adv Powder Technol 4:79-101.

Bezrukov A, Stoyan D, Bargiel M (2001). Spatial statistics for simulated packings of spheres. Image Anal Stereol 20:203-6.

Bezrukov A, Bargiel M, Stoyan D (2002). Statistical analysis of simulated random packings of spheres. Part Past Syst Char 19:111-8.

Binder K (1997). Applications of Monte Carlo methods to statistical physics. Rep Prog Phys 60:487-559.

Binder K (2001). How Monte Carlo simulations can clarify complex problems in statistical physics. Int J Mod Phys B 12:1193-211.

Cundall PA (1971). A computer model for simulating progressive large scale movements in blocky rock systems. In: Proc Symp Int Soc Rock Mechanics, Nancy, France, Vol. 1, Paper No. II 8. 
Cundall PA, Hart R. (1992). Numerical modeling of discontinua. J Engr Comp 9:101-13.

Delarue A, Jeulin D (2001). Multi-scale simulation of spherical aggregates. Image Anal Stereol 20:181-6.

Döge G (2001). Perfect simulation for random sequential adsorption of $d$ dimensional spheres with random radii. J Statist Comput Simul 69:141-56.

Evans JW (1993). Random and cooperative sequential adsorption. Rev Modern Phys 65:1281-304.

Gilks WR, Richardson S, Spiegelhalter DJ (1996). Markov Chain Monte Carlo in Practice. London: Chapman and Hall.

Hastings WK (1970). Monte Carlo sampling methods using Markov chains and their applications. Biometrika 57:97-109.

Jodrey WS, Tory EM (1979). Simulation of random packing of spheres. J Simulation 32:1-12.

Jodrey WS, Tory EM (1985). Computer simulation of close random packing of equal spheres. Phys Rev A 32:234751 .

Marinari E, Parisi G (1992). Simulated tempering: A new Monte Carlo scheme. Europhysics Letters 19:451-8.

Mase S, Møller J, Stoyan D, Waagepetersen RP, Döge G (2001). Packing densities and simulated tempering for hard core Gibbs point proceses. Ann Inst Statist Math 53:661-80.

Mecke J, Stoyan D (2001). The specific connectivity number of random networks. Adv Appl Prob 33:576-83.

Mecke K, Wagner H (1991). Euler characteristics and related measures for random geomeric sets. J Statist Phys 64:843-50.

Mecke K (2000). Additivity, convexity, and beyond: Applications of Minkowski functionals in statistical physics. In: Mecke KR, Stoyan D, eds. Statistical Physics and Spatial Statistics. Springer Lecture Notes in Physics 554:111-84.
Metropolis N, Rosenbluth AW, Rosenbluth MN, Teller AH, Telle E (1953). Equations of state calculations by fast computing machines. J Chem Phys 21:1087-92.

Moscinski J, Bargiel M, Rycerz ZA, Jacobs PWM (1989). The force biased algorithm for the irregular close packing of equal hard spheres. Molecular Simulation $3: 201-12$.

Schmid F (2002). Computer simulations of systems of ellipsoids. In: Mecke KR, Stoyan D, eds. Morphology of Condensed Matter. Physics and Geometry of Spatially Complex Systems. Springer Lecture Notes in Physics.

Stillinger FH, DiMarzio EA, Kornegay RL (1964). Systematic approach to explanation of the rigid disk phase transition. J Chem Phys 40:1564-76.

Stoyan D, Kendall WS, Mecke J (1995). Stochastic Geometry and its Applications. Chichester: J Wiley and Sons.

Stoyan D, Schlather M (2000). Random sequential adsorption: relationship to dead leaves and characterization of variability. J Statist Phys 100:96979.

Stoyan D, Stoyan H (1994). Fractals, Random Shapes and Point Fields. Chichester: J. Wiley and Sons.

Torquato S (1991). Random heterogeneous media: Microstructure and improved bounds on effective properties. Appl Mech Rev 44:37-76.

Torquato S (2002). Random Heterogeneous Materials. New York: Springer-Verlag.

Tory EM, Church BH, Tam MK, Ratner M (1973). Simulated random packing of equal spheres. Canad J Chem Eng 51:484-93.

Truskett TM, Torquato S, Sastry S, Debenedetti PG, Stillinger FH (1999). A structural precursor to freezing in the hard disk and hard sphere systems. Phys Review E 58:3083-8. 